\title{
CRITICAL CURRENT DEGRADATION IN Nb3Sn CABLES UNDER TRANSVERSE PRESSURE
}

\author{
H.H.J. ten Kate, H.W. Weijers, J.M. van Oort ${ }^{\#}$ \\ Applied Superconductivity Center, University of Twente, \\ P.O. Box 217, 7500 AE Enschede, \\ The Netherlands. \\ (\#) Lawrence Berkeley Laboratory, USA.
}

\begin{abstract}
The critical current degradation of a few Rutherford type of $\mathrm{Nb}_{3} \mathrm{Sn}$ cables are investigated as function of transverse pressure. Moreover a comparison is made between $\mathrm{Nb}_{3} \mathrm{Sn}$ strands produced according to the Powder-in-Tube, the Bronze and the Modified Jelly Roll process. The (keystoned) Rutherford cables are charged at $11 \mathrm{~T}$ with transverse pressures up to $250 \mathrm{MPa}$. Large differences in critical current reduction are observed, ranging from 6 to about $60 \%$ at $200 \mathrm{MPa}$ depending on the type of $\mathrm{Nb}_{3} \mathrm{Sn}$. It appears that the irreversible part is about $40 \%$ of the total reduction. Moreover, the "irreversible" part shows relaxation and a partial recovery is possible by thermal cycling.
\end{abstract}

\section{INTRODUCTION.}

The generation of magnetic fields beyond 10 tesla requires the application of $\mathrm{Nb}_{3} \mathrm{Sn}$ cables which material in general is characterized as very sensitive to mechanical deformations. Therefore, in the framework of the development of an experimental $11.5 \mathrm{~T}$ LHC type of dipole magnet $([1])$, a study of the transverse stress effects on $\mathrm{Nb}_{3} \mathrm{Sn}$ was started since in these magnets stress levels up to $150 \mathrm{MPa}$ can be expected. Further on a collaboration with LBL was set up in connection to the LBL development project for their $13 \mathrm{~T}$ magnet D20.

The program is carried out along three routes:

* study of the basic stress tensor by investigating a simple 2 dimensional $\mathrm{Nb}_{3} \mathrm{Sn}$ layer or tapes ([2]),

* experiments on strand materials ([3]), and

* the investigation of complex cables $([4,5])$, which will be dealt with in this paper.

The execution of these routes in parallel should yield a better understanding of the basic degradation mechanism. The electric field $E$ along the superconductor is a well known function of the current, the field and the temperature but the additional effect of the strain tensor has still to be clarified in detail.

A lot is known about the effect of axial stress on superconducting wires and even the effect of transverse stress on single wires has been studied by many authors $([3,6])$. However experimental data about cables are

This work was supported in part by FOM, the Netherlands Foundation for Fundamental Research on Matter, Utrecht, the Netherlands. Manuscript received August 24, 1992. scarce $([4,5,7])$. It is the aim of our research to investigate the behaviour of Rutherford cables under realistic operation conditions and to study the reproducibility of the results as well as to learn about the differences between various types of $\mathrm{Nb}_{3} \mathrm{Sn}$. Especially for this a test facility was developed and during the last 2 years 11 cable pieces have been investigated.

\section{TEST FACILITY}

The test facility consist of an a $16 \mathrm{~T} / 80 \mathrm{~mm}$ magnet in combination with a current supply and a cryogenic press. The current supply is a superconducting transformer system operated in a feedback mode to generate a truly stationary current in the sample of $50 \mathrm{kA}$ maximum. The press, capable of producing $250 \mathrm{kN}$ which is equivalent to about $300 \mathrm{MPa}$ transverse pressure onto the cable, also is a special development. It consists of a superconducting coil system by which the repulsing force between the coils is transferred to the pressure blocks which impresses a prepared section of the cable. The force put on the cable can be easily adjusted by control of the current in the coil system.

The cable to be investigated is formed into a U-shape. The legs are in parallel field and connected to the current supply. In the intermediate section which is in transverse field, the pressure is applied across $40 \mathrm{~mm}$ of conductor, see Fig. 1. Both the field homogeneity and the accuracy of the current measurement are better then $1 \%$. The uncertainty in the applied pressure is about $2 \%$.

Several $\mathrm{nV}$-meters (Keithley 181/182) are used to measure sample voltages. In combination with the ripple free sample current, very accurate voltage-current measurements on the cables can be performed with a resolution of about $50 \mathrm{nV}$. More details are in ([5]).

\section{THE SAMPLES}

Results obtained with 7 new samples are presented while the former 4 measurements are memorized. The main parameters of the cables are given in Table 1 .

Cable (1), made by ECN in the Netherlands of wires produced according to the Powder-in-Tube (PT) method ([8]), was used by CERN for the construction of an experimental LHC model mirror magnet.

Cable (2) is also made by ECN and it was used for magnets in the EURATOM SULTAN project. 


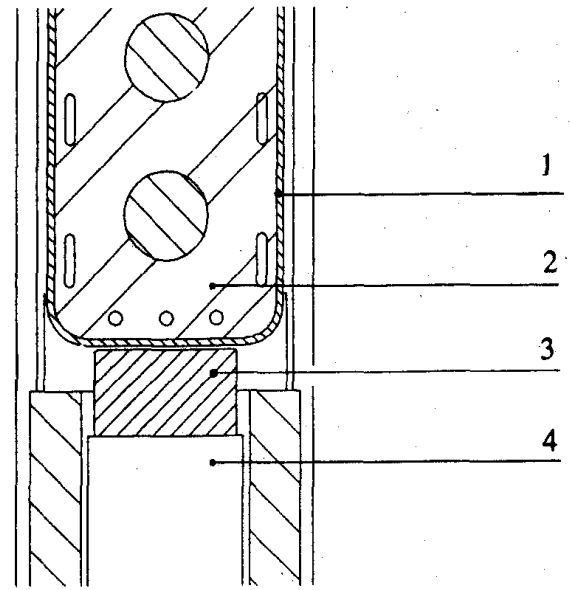

Fig. 1 The central part of the pressing arrangement:

(1) U shape cable, (2) sample holder,

(3) pressure block, (4) pressure pin.

Cables (3) and (4) are experimental conductors made by LBL of Modified Jelly Roll (MJR) wires of Teledyne Wah Chang Albany. Note that cable (4) has strands with 6 sub elements which is a relatively old conductor.

Cable (5), made by Vacuumschmelze in Germany according to the Bronze Route (BR), was applied by CERN and ELIN in an LHC model magnet.

All samples are prepared and measured using the same techniques and equipment. A few cables were tested a few times to check reproducibility. The samples are marked by an abbreviation indicating the production technique preceded by a serial number. For the MJR samples also the number of strands is indicated.

The heat treatments of the cable were as specified:

ECN PT: 64 hrs. at $675^{\circ} \mathrm{C}$; VAC-BR: 144 hrs. at $650^{\circ} \mathrm{C}$; TWCA-MJR: $100 \mathrm{hrs}$. at $200^{\circ} \mathrm{C}, 24 \mathrm{hrs}$. at $340^{\circ} \mathrm{C}$,

$48 \mathrm{hrs}$. at $580^{\circ} \mathrm{C}, 48 \mathrm{hrs}$. at $650^{\circ} \mathrm{C}$.

Except 1PT, all the samples were impregnated with resin (STYCAST2850FT). Sample 1PT was covered only partly.

\section{RESULTS.}

The cables 1 and 2 were investigated before. Sample 1PT showed a severe degradation of about $50 \%$ at $100 \mathrm{MPa}$ caused by a bad impregnation ([4]). Moreover the pressure block was in direct contact with the cable surface which prohibited a uniform pressing of the cable. Therefore, also samples 2PT and 3PT gave rather large degradations of about $25 \%$ at $200 \mathrm{MPa}$. After some improvements the Ic reductions were lower and became reproducible. The Ic degradation of the fourth sample $4 \mathrm{PT}$ is $3 \%$ at 100 and $9 \%$ at $200 \mathrm{MPa}$ respectively ([5]), which data are confirmed by the result of sample 5PT.

\section{A. The shape of the voltage-current curves}

The voltage of several individual strands are measured as well as the global voltage of the cable by which the taps cover the entire cable section. A nice example of the differences are shown in Fig. 2, in which also the Ic criterion lines $5 \mathrm{e}-14,1 \mathrm{e}-13$ are indicated. It was not possible to correlate the voltages of strands with their position in the cable. For example, taps \#4 showing the lowest voltage is a strand which lies at the thin edge of the cable where one would expect the highest voltage. Note that the global contact gives about the average voltage and at a level of $5 \mu \mathrm{V}$ all taps give the same critical current.

Further conclusions are that the spread of the voltages slightly increases at higher pressures but the sequence is not changed. This means that the applied pressure is about uniform. Moreover is has been proven that the self field and the direction of the Lorentz force on the test section do not effect the U-I behaviour within the measuring accuracy.

In Fig. 3 an example is given of the U-I curves as function of the applied transverse stress. The nice shifting of the voltage for increasing stress can be recognized. Further examination of these curves for all

Table 1. Characteristics of the $\mathrm{Nb}_{3} \mathrm{Sn}$ Rutherford type of cabled conductors.

\begin{tabular}{|c|c|c|c|c|c|}
\hline Type: & (1) ecn lhc-b & (2) ecn sultan & twca/lbl (3) & twca/lbl (4) & vac-lhc-a (5) \\
\hline Production: & powder in tube & powder in tube & MJR 26 & MJR 48 & bronze route \\
\hline Model: & keystoned & rectangular & keystoned & keystoned & keystoned \\
\hline Dimensions [mm]: & $(1.53 / 1.85) 16.6$ & $(1.82 / 1.82) 18.2$ & $(2.21 / 2.51) 17.0$ & $(1.07 / 1.29) 15.8$ & $(2.19 / 2.69) 16: 8$ \\
\hline Number of strands: & 36 & 36 & 26 & 48 & 24 \\
\hline Number of filaments: & 192 & 192 & 6 subelements & 36 subelements & 50000 \\
\hline Diameter strand [mm]: & 0.90 & 1.00 & 1.29 & 0.65 & 1.38 \\
\hline Matrix: & $\mathrm{Cu}$ & $\mathrm{Cu}$ & $\mathrm{Cu} / \mathrm{CuSn} / \mathrm{barr}$. & $\mathrm{Cu} / \mathrm{CuSn} / \mathrm{barr}$. & $\mathrm{Cu} / \mathrm{CuSn} / \mathrm{barr}$ \\
\hline$\% \mathrm{Cu}[\%]$ & 57 & 55 & 48 & 56 & 29 \\
\hline Pitch cable $[\mathrm{mm}]$ & 120 & 160 & 119 & 109 & 150 \\
\hline Measured Ic@11T [kA] & :16.5 & 19.6 & 9.2 & 3.3 & 15.1 \\
\hline Samples: & $1 \mathrm{PT}$ & $2,3,4,5$ PT & 7,8,9 MJR26 & 6 MJR48 & $10,11 \mathrm{BR}$ \\
\hline
\end{tabular}






Fig. 2 Example of the spread in voltages measured on strands in the cable. \#1=global taps, \#2,3,4,5 are various strands under the pressure block.

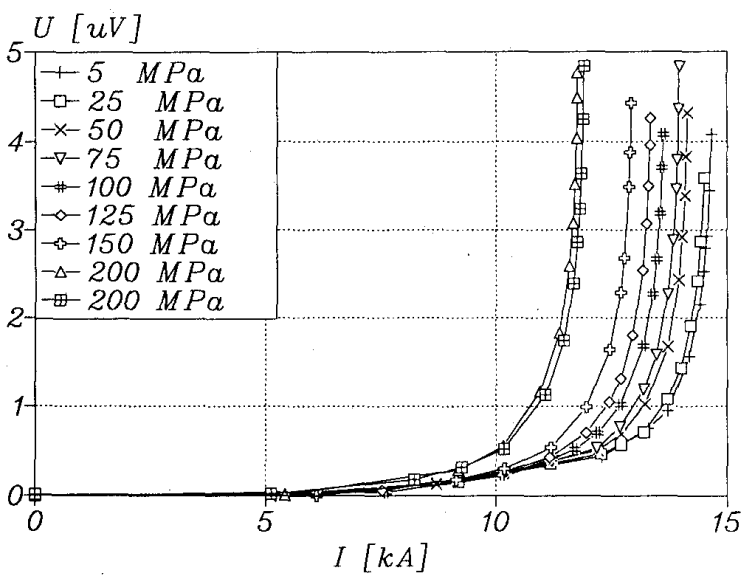

Fig. $3 \mathrm{U}$-I curves at $11 \mathrm{~T}$ and a stress of 5-200 MPa. $0<\mathrm{U}<5 \mu \mathrm{V}, 0<\mathrm{I}<15 \mathrm{kA}$. Sample 10BR (VAC).

samples has yielded the following conclusions.

The U-I curves can not be described by the n-power law $U(I)=$ constant $\times I^{n}$, as usually applied satisfactory in the case of monolithic wires. For example, in the case of Fig. 3, the $n$ value is 4 at $10 \mathrm{kA}, 5 \mathrm{MPa}$ and increases to 40 at the voltage level of $5 \mu \mathrm{V}$.

The applied stress does not influence the current sharing part of the U-I curve but only the superconducting part. The shape of the transition remains within the available accuracy unchanged.

\section{B. Critical current versus strain, summary of results}

The main differences between the three types of $\mathrm{Nb}_{3} \mathrm{Sn}$ are presented in Fig. 4 in which the critical current reduction (applying the $1 \mu \mathrm{V} / \mathrm{cm}$ criterion) is

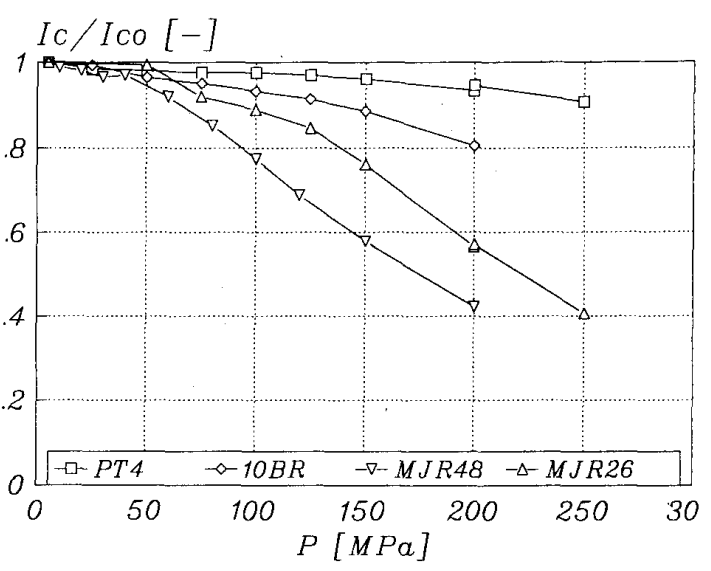

Fig. 4 Scaled critical current versus the applied transverse stress for several cable samples.

given as function of the applied transverse stress up $250 \mathrm{MPa}$. This picture is based on the samples 5-12. Within the measuring error of about $2 \%$ the results have been reproduced by second samples. The conclusions that can be drawn from the picture are collected in Table 2 .

Table 2. Summary critical current reductions vs stress.

\begin{tabular}{|c|c|c|}
\hline $\mathrm{B}=11 \mathrm{~T}$. and $1 \mu \mathrm{V} / \mathrm{cm}$ criterion: & $100 \mathrm{MPa}$ & $200 \mathrm{MF}$ \\
\hline ECN type powder-in-tube: & $2-4 \%$ & $5-8 \%$ \\
\hline VAC type bronze route: & $7-10 \%$ & $18-22 \%$ \\
\hline TWCA MJR route, 6 subelement & $: 20-25 \%$ & $55-60 \%$ \\
\hline ,36 subelements: & $10-12 \%$ & $40-45 \%$ \\
\hline
\end{tabular}

It appears that the PT conductor, characterized by the thick $\mathrm{Nb}$ tubes that enclose the $\mathrm{Nb}_{3} \mathrm{Sn}$ layers, shows the best strain resistance. The MJR wires as far as tested here are extremely sensitive to stress. Especially the "old" conductor with 6 sub elements shows a severe degradation. It is recommended to investigate the more recently produced MJR wires with 120 and 378 sub elements to check possible improvements.

All samples are also investigated at $9 \mathrm{~T}$. The qualitative behaviour is the same but the critical reductions at $9 \mathrm{~T}$ are about $75 \%$ of those at $11 \mathrm{~T}$.

Sample 8MJR26 is tested with a pressure block not matching the keystone angle of the cable. In this case the pressure is not uniform and consequently the measured degradations are 50 and $90 \%$ at 100 and $200 \mathrm{MPa}$ respectively. Also the irreversible part of the Ic degradation has increased compared to the normal case.

\section{Relaxation of the "irreversible" degradation}

Another important observation concerns the "ir- 
when the applied stress is made zero again. It appears that the irreversible part after applying a certain stress amounts to $30-50 \%$ of the actual degradation under stress. This is valid for all the samples 4-11.

However by chance it was discovered that in the case of sample 11BR the irreversible part is not persistent. After thermal cycling, i.e. a repeat of the measurement after 15 days and a warming up to room temperature, the critical current of this particular sample has attained its virgin value again (even a little bit more). In Fig. 5 this "relaxation" is demonstrated for the 11BR sample. After an interval and a

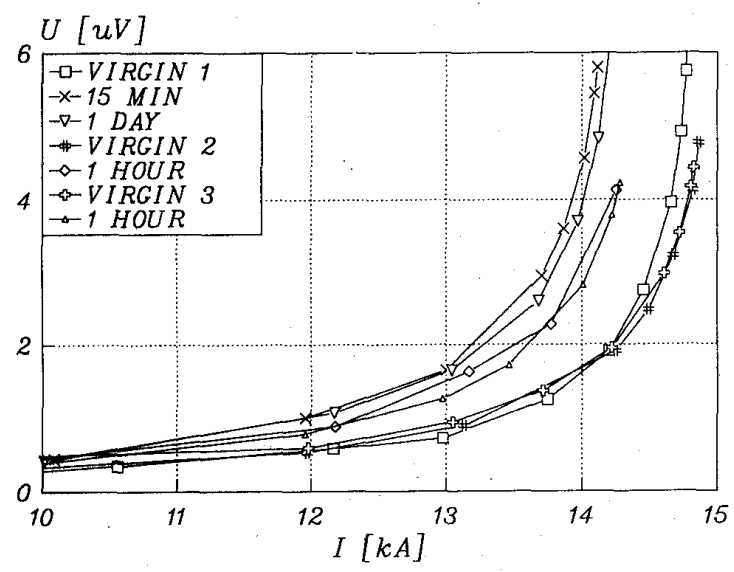

Fig. 5 U-I curves of sample 11BR showing relaxation of the "irreversible" part of the critical current.

thermal cycle the virgin critical current could be restored. This observation proves that (at least) in the BR sample up to $200 \mathrm{MPa}$, no permanent damage has occurred. To check this the filaments in the sample have been examined with SEM and it appeared that there is no difference in the small number of interrupts as present in unpressed and pressed strands.

Obviously the Ic reduction in the BR sample of $20-25 \%$ is completely reversible by relaxation and consequently the Ic reduction under transverse stress of $200 \mathrm{MPa}$ is not caused by damage to the $\mathrm{Nb}_{3} \mathrm{Sn}$ filaments. Quite a different behaviour occurs in the MJR conductor: The irreversible reduction is 20 to $50 \%$ and is persistent. A SEM micro analysis of the strands clearly shows visible damage ([9]).

\section{CONCLUSIONS}

For the first time a systematic study of the Ic reduction of impregnated $\mathrm{Nb}_{3} \mathrm{Sn}$ Rutherford cables caused by transverse stress has been carried out. Remarkable and reproducible differences between three types of
$\mathrm{Nb}_{3} \mathrm{Sn}$ are found.

At a stress level of $200 \mathrm{MPa}$ Ic reductions of about 7, 20 and 40-60\% are found for the Powder in Tube, the Bronze type and the Modified Jelly Roll conductors respectively.

About $40 \%$ of the Ic reduction under stress is "irreversible". It is found for the first time (in one sample) that in principle relaxation of the "irreversible" part can occur by thermal cycling. In that case also no visible additional damage to the $\mathrm{Nb}_{3} \mathrm{Sn}$ filaments due to applying the transverse pressure is found.

The major part of the actual Ic reduction is determined very strongly by the internal lay-out of the filaments and the material composition of the conductor. In the (old) MJR conductors a lot of cracks are observed and it correlates with a large irreversible (when cold) and permanent (in time) degradation. On the other hand, the bronze conductor shows a moderate Ic reduction and after thermal cycling the virgin critical current could be restored.

\section{REFERENCES}

[1] H.H.J. ten Kate, e.a.,"Development of an experimental $10 \mathrm{~T} \mathrm{Nb}_{3}$ Sn Dipole magnet for the CERN LHC", IEEE, Tr, on Magn., MAG-27, 2. pp,1996, 1991.

[2] B. ten Haken, A.E. Reitsma, H.H.J. ten Kate, "The reduction of the intrinsic critical current density dueto parallel strain in $\mathrm{Nb}_{3} \mathrm{Sn}$ tape like con ductors", in Proc. ICEC-14, Kiev, GOS, June 1992.

[3] H. Boschman, H.H.J. ten Kate, L.J.M. van de Klundert, "Voltage-currentt characteristics of multifilamentary $\mathrm{Nb}_{3} \mathrm{Sn}$ wires under transverse, compres sive stress", Proc. ICEC-13, Beijing, China, 1990.

[4] H. Boschman, A.P. Verweij, S. Wessel, H.HJ ten Kate, L.M.J. van de Klundert, "The effect of transverse loads up to $300 \mathrm{MPa}$ on the critical currents of $\mathrm{Nb}_{3} \mathrm{Sn}$ cables", IEEE MAG-27, 1991.

[5] H.H.J, ten Kate, e.a.,"The reduction of the criti cal current in $\mathrm{Nb}_{3} \mathrm{Sn}$ cables under transverse for ces", Proc. MT-12, Leningrad, 1991.

[6] J.W. Ekin, "Effect of transverse compressive stress on the critical current and upper critical field of $\mathrm{Nb}_{3} \mathrm{Sn}$ ", J. of Appl. Phys., vol 62, no 12, pp. $4829-4834,1987$.

[7] B. Jakob, e.a. "Reduced sensitivity of $\mathrm{Nb}_{3} \mathrm{Sn}$ epoxy-impregnated cable to transverse stress", Cryogenics 1991.

[8] E.M. Hornsveld, e.a. "Development of ECN-type of Niobium Tin wire towards smaller filament size", Adv. in Cryogenic Eng, vol 34, pp. 493-498, 1987

[9] J.M. van Oort, R.M. Scanlan, H.W. Weijers, S. Wessel, H.H.J. ten Kate, "The reduction of the critl cal current in $\mathrm{Nb}_{3} \mathrm{Sn}$ cables under transverse loads", Proc. ASC 1992, Chicago, 1992, in print 